\title{
Protoplast Fusion as a Tool for Genetic Analysis in Cephalosporium acremonium
}

\author{
By PAUL F. HAMLYN, $\dagger^{+}$J. A. BIRKETT, ${ }^{*}$ GASPAR PEREZ $^{1}$ AND \\ JOHN F. PEBERDY ${ }^{1}$ \\ 'Microbial Genetics and Biochemistry Laboratory, Department of Botany, \\ University of Nottingham, Nottingham NG7 2RD, UK \\ 2 Glaxochem Limited, Ulverston, Cumbria LA12 9DR, UK
}

(Received 27 November 1985; revised 6 May 1985)

\begin{abstract}
Protoplasts of nutritionally complementary strains of Cephalosporium acremonium were fused and plated onto media which supressed the growth of both parents. The regenerating colonies were used for genetic analysis and were found to be of two types, stable haploid recombinants and unstable heterozygotes (aneuploids and/or diploids). Analysis of these colonies provided evidence for eight linkage groups and a relatively high rate of mitotic crossing-over. The gene order for three of the markers on one linkage group was also determined.
\end{abstract}

\section{INTRODUCTION}

Cephalosporium acremonium (syn. Acremonium chrysogenum Gams) has been of considerable commercial importance for nearly two decades. Newton \& Abraham (1956) discovered that the organism could synthesize a novel, penicillinase resistant, $\beta$-lactam antibiotic, cephalosporin $\mathrm{C}$. C. acremonium is an imperfect species; attempts to carry out genetical analyses or selective breeding using classical parasexual techniques were frustrated by the difficulty in obtaining heterokaryons or diploids (Nuesch et al., 1973; Elander et al., 1976; Hamlyn \& Ball, 1979). These difficulties may stem from the fact that the organism has uninucleate hyphal compartments which could restrict heterokaryosis and subsequent nuclear mixing. Protoplast fusion (Peberdy, 1979, 1980; Ferenczy, 1981) offered a method of bringing together nuclei from different strains of $C$. acremonium in a common cytoplasm. The unexpected discovery by Hamlyn \& Ball (1979) that recombinant progeny could be isolated directly from fusion plates suggested that diploid strains of C. acremonium were very unstable. This view has gained further support from the work of others who have failed to isolate stable diploids (Chang et al., 1982; Minuth \& Esser, 1983).

The aim of the present work was to determine if the analysis of protoplast fusion products could provide sufficient data to undertake genetic mapping studies. If successful it would generate basic genetic data on a commercially important organism and extend the knowledge of variations in the parasexual process of fungi.

\section{METHODS}

Strains and culture conditions. The strains of $C$. acremonium used in these experiments were all derived from strain M-8650, which was obtained from Glaxochem Ltd, Ulverston, UK ; their genotypes and origins are listed in Table 1. The following allele codes and phenotypes are used in the paper: ane, arg, ino, leu, lys and met are requirements for aneurin, arginine, inositol, leucine, lysine and methionine respectively; azu, ben and pyt are resistance to 6-azauracil, benlate and pyrithiamine respectively; mor is abnormal colony morphology. Cultures were maintained on complete medium and grown at $25^{\circ} \mathrm{C}$.

† Present address: Shirley Institute, Didsbury, Manchester M20 8RX, UK. 


\section{Table 1. Strains and their origins}

For strains originating through mutation the markers are listed in the order of introduction; arg- $I$ and mor- $l$ were introduced simultaneously by the same treatment. UV light was used as the mutagen, except for $a z u-l$ and ben-l, which were isolated as spontaneous mutations.

Strain genotype

\author{
ane-5 ino-1 \\ lys-1 his-1 \\ azu-1 arg-2 met-1 \\ azu-l arg-1 mor-1 leu-1 red-1 pyt-1 \\ azu-I arg-1 mor-1 leu-1 red-1 pyt-1 ben-1 \\ pyt-1 red-1 lys-1 \\ pyt-l ino-l arg-l azu-l mor-l ben-l \\ leu-1 ino-I mor-1 ben-1 \\ pyt-1 red-1 arg-2 \\ red-1 ino- 1 azu-1 ben-1
}

\section{Origin}

\author{
Mutation \\ Mutation \\ Mutation \\ Mutation \\ Mutation \\ Recombinant, cross 1 \\ Recombinant, cross 2 \\ Recombinant, cross 2 \\ Recombinant, cross 4 \\ Recombinant (Hamlyn, 1982)
}

Media. Complete medium (CM) contained $\left(\mathrm{g}^{-1}\right)$ : maltose, 40 ; Oxoid peptone, 10; Oxoid malt extract, 24; agar, 20. It was adjusted to $\mathrm{pH} 7.5$ and then autoclaved at $121^{\circ} \mathrm{C}$ for $20 \mathrm{~min}$. Minimal medium (MM) contained $\left(\mathrm{g} \mathrm{l}^{-1}\right)$ : glycerol, $30 ; \mathrm{NaNO}_{3}, 2 ; \mathrm{KH}_{2} \mathrm{PO}_{4}, 1 ; \mathrm{MgSO}_{4} .7 \mathrm{H}_{2} \mathrm{O}, 0.5 ; \mathrm{KCl}, 0.5 ; \mathrm{FeSO}_{4} .7 \mathrm{H}_{2} \mathrm{O}, 0 \cdot 01 ;$ Difco Bacto agar, 20. It was adjusted to $\mathrm{pH} 6.8$ and then autoclaved at $121^{\circ} \mathrm{C}$ for $20 \mathrm{~min}$. MM was supplemented, as appropriate, with amino acids, $100 \mu \mathrm{g} \mathrm{ml}^{-1}$; vitamins, $10 \mu \mathrm{g} \mathrm{ml}^{-1}$; 6-azauracil, $500 \mu \mathrm{g} \mathrm{ml}^{-1}$; benlate, $2.5 \mu \mathrm{g} \mathrm{ml}^{-1}$; pyrithiamine, $10 \mu \mathrm{g} \mathrm{ml}^{-1}$. Hypertonic minimal medium (SMG) used for protoplast regeneration was MM supplemented with $0 \cdot 7 \mathrm{M} \cdot \mathrm{NaCl}$ as osmotic stabilizer.

Protoplast preparation and fusion. Protoplasts were isolated as described previously (Hamlyn et al., 1981) using cellulase CP (John \& E. Sturge Ltd, Selby, UK) as the lytic enzyme and $0.7 \mathrm{M}-\mathrm{NaCl}$ as osmotic stabilizer. Fusion of protoplasts was done using polyethylene glycol (mol. wt 6000; Sigma) (PEG), by a similar method to that described by Anne \& Peberdy (1976) and Ferenczy et al. (1975).

Protoplasts were separated from mycelial debris by slow centrifugation and washed twice in $0 \cdot 7 \mathrm{M}-\mathrm{NaCl}$. An equal number of protopiasts from each strain $\left(10^{8} \mathrm{of}\right.$ each) were mixed and centrifuged ( $700 \mathrm{~g}$ for $\left.10 \mathrm{~min}\right)$. The pellet was then resuspended in $2 \mathrm{ml} \mathrm{30 \% (w/v)} \mathrm{PEG,} \mathrm{containing} 0.01 \mathrm{M}-\mathrm{CaCl}_{2}$ and $0.05 \mathrm{M}$-glycine (adjusted to $\mathrm{pH} 7.5$ with $\mathrm{NaOH}$ and sterilized by membrane filtration), and incubated for $10 \mathrm{~min}$ at $28{ }^{\circ} \mathrm{C}$. Finally, the suspension was diluted with $8 \mathrm{ml} 0.7 \mathrm{M}-\mathrm{NaCl}$ and centrifuged $(700 \mathrm{~g}$ for $10 \mathrm{~min}$ ). Treated protoplasts were washed twice with $0.7 \mathrm{M}-\mathrm{NaCl}$ and then resuspended in $10 \mathrm{ml} 0.7 \mathrm{M}-\mathrm{NaCl}$.

Isolation of segregants and genetic analysis. PEG-treated protoplast suspensions were plated undiluted by spreading $0.1 \mathrm{ml}$ onto cellophane discs on SMG agar plates. The medium was partially supplemented with the growth requirements of the parental strains to allow the recovery of recombinant segregants. Thus for a threefactor cross, e.g. $\mathrm{Lys}^{-} \times \mathrm{Arg}^{-} \mathrm{His}^{-}$, recombinants were selected on medium containing arginine and on medium containing histidine. Non-selective markers such as mycelial pigmentation and resistance to metabolic inhibitors were also employed in these crosses. Control plates were inoculated to check for back mutation of auxotrophic markers and also to determine the total number of viable protoplasts after fusion. After $4 \mathrm{~d}$ incubation at $25^{\circ} \mathrm{C}$ the cellophane discs were transferred to fresh medium lacking the osmotic stabilizer and incubated for a further 6 $10 \mathrm{~d}$. Generally two broad groups of colony type were observed; large fast growing forms, which proved to be haploid recombinants, and smaller slower growing more compact colonies, which were found to be heterozygotes. Random colonies were taken from each medium and purified by plating fragmented mycelium onto $\mathbf{M M}$ containing the appropriate supplements. In practice, each colony was picked off, transferred into a tube of sterile water and thoroughly mixed on a vortex mixer for $30 \mathrm{~s}$ to give a suspension of small hyphal fragments. This was necessary because the organism sporulated very poorly on these media. Single colony isolates were subsequently characterized by replication to the appropriate diagnostic media.

Heterozygotes were recognized from the fact that on subculture they yielded a mixture of normal and small abnormal types. Analysis was done by macerating the small abnormal colonies, plating the fragments onto CM and allowing the colonies generated by this procedure to produce outgrowths (sectors). A single sector was taken from each unstable colony, purified and characterized as if it had been isolated from a fusion plate. By minimizing the growth period and using more than one heterozygotic colony from the first subculture after fusion, it was possible to reduce clonal effects. 


\section{RESULTS AND DISCUSSION}

\section{Enhancements to the recovery techniques}

The original techniques used for the recovery of fusion products (Hamlyn \& Ball, 1979) proved inappropriate with certain combinations of auxotrophic strains. In such cases the fusion plates were covered by fast growing colonies resembling heterokaryons. It is unlikely that these colonies were true heterokaryons because they failed to grow when subcultured onto normal MM. They were probably formed by syntrophic growth, resulting from the leakage of metabolites on hypertonic media, after protoplast fusion had provided the initial stimulus. The problem was reduced by substituting glycerol for sucrose in the regeneration medium and by plating the fused protoplasts onto cellophane discs. Transferring these discs to normal media after the fusion products had regained osmotic stability reduced the adverse effects of growth under hypertonic conditions.

\section{Analysis of haploid selectants}

In the majority of cases the fusion products recovered from the cellophane discs were haploid recombinants. The ploidy was inferred from their stability in the presence of haploidizing agents and spontaneous mutation rates; confirmation came from DNA measurements (Hamlyn, 1982).

The ability to isolate haploid recombinants directly after protoplast fusion offers a potentially powerful technique for genetical analysis; unfortunately its full potential can rarely be realised because environmental effects distort the data. Certain mutant alleles proved to be less fit than their wild-type counterpart and were selectively lost during protoplast regeneration. The problems were overcome by approaching the analyses in a qualitative rather than quantitative manner. This initial study used a variety of mutant and recombinant strains to investigate the general applicability of the techniques. Two types of selective cross were done; one used four selective markers and four selective media, the other used three selective markers and two media. These were termed four- and three-factor crosses, respectively.

\section{Four-factor crosses}

Cross 1, azu-1 arg-1 mor-1 leu-1 red-1 pyt-1 $\times$ lys-1 his-1, involved two strains differing in at least seven mutant alleles. Recombinants were obtained on all of the selective media (Table 2) and the results illustrate many of the effects encountered in these analyses. Two cases of tight linkage were found, red-l to his-l and lys-l to $a z u-I$; since both involved selective markers the unselected markers showed uneven segregation frequencies on the different media. The segregation frequencies of the $l e u-I$ and $l y s-l$ alleles were also unequal (Table 3 ) but this reflected an under-recovery of the mutant allele through reduced fitness rather than linkage. Other abnormal frequencies probably resulted from sampling error effects.

Cross 2, azu-l arg-1 mor-1 leu-1 red-1 pyt-1 ben-1 $\times$ ane-5 ino-1, was similar to cross 1 , and utilized eight allelic differences, five of which were common to cross 1 . Two further cases of tight linkage (Table 4) were observed; red- $I$ to ino- $I$ and ben- $I$ to ane-5. The leu- $I$ allele was again at a selective disadvantage and on all four media there were apparent sampling error effects. The latter resulted from a preponderance of two particular genotypes, red- 1 ane- $I$ and ben- 1 ino- 1 ; they accounted for $25 \%$ of the segregants on the arginine-containing media and $50 \%$ on the leucine media. There were no obvious reasons for the increased fitness of these two recombinant types.

The third four-factor cross involved a recombinant from cross 2, pyt-1 ino-1 arg-1 azu-1 ben-1, and the his-1 lys-1 strain from cross 1 . This cross was characterized by an inability to suppress syntrophic growth on two of the media. The results from crosses 1 and 2 predicted that his-l and ino- $l$ should be linked; this was confirmed, as was the linkage of $l y s-l$ and $a z u-l$. As with cross 1 there was a selective loss of lys- 1 progeny.

Using these three crosses it had been possible to test eleven markers against each other, with the exception of mor-1, which had not been tested against red-1, leu- 1 or ane-5; two of these three had been tested indirectly through their linkage to other markers. The data from these three 
Table 2. Results from the analysis of selectants from cross 1 : azu-1 arg-1 mor-1 leu-1 red-1 pyt-1 $\times$ lys-1 his-1

Values are numbers of colonies; those given in italics denote colonies with recombinant genotypes. + , Wild-type allele; -, mutant allele; *, genotype could not be recovered on that medium. mor- 1 was not scored amongst these selectants.

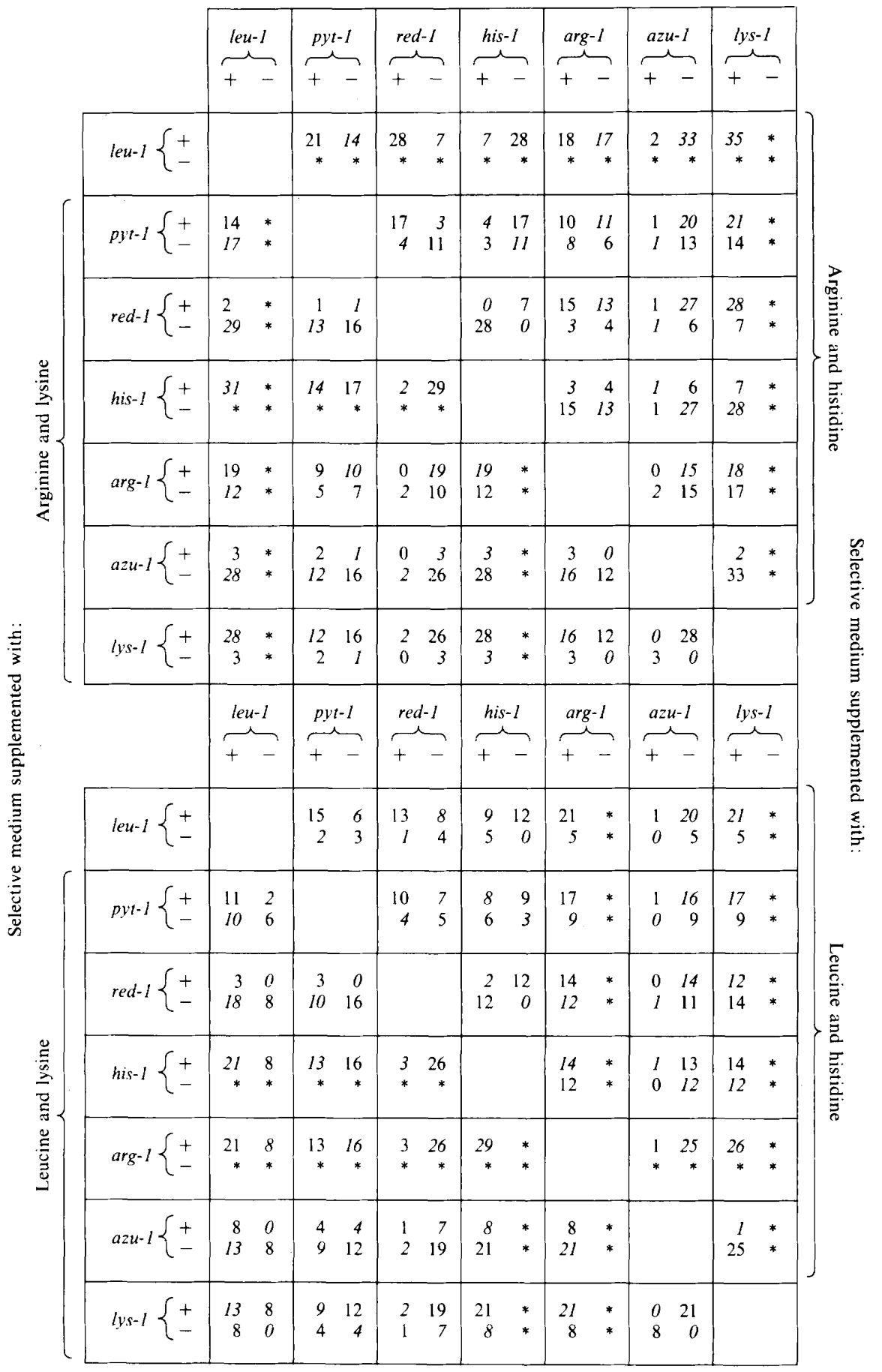


Table 3. Behaviour of the auxotrophic markers in cross 1: azu-1 arg-1 mor-1 leu-1 red-1 pyt $-1 \times$ lys-1 his -1

Selective medium supplemented with:

Arginine and histidine Arginine and lysine Leucine and histidine Leucine and lysine

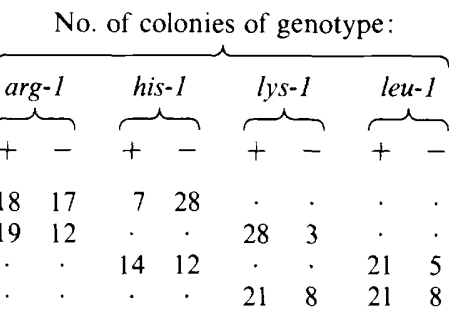

+ , Wild-type allele; -, mutant allele.

crosses suggested that there was a minimum of six linkage groups. The questions left unanswered were how did the new data match that of Hamlyn \& Ball (1979), and what was the relationship between the three linked markers, red-1, ino- 1 and his-1? These questions were approached through the use of three-factor crosses, which were easier to handle and less demanding in terms of strain construction.

\section{Three-factor crosses}

Several crosses were set up to determine the relationship between met-1, arg- 2 and the other markers. Cross 4, azu-1 arg-2 met-1 $\times$ pyt-I red-1 lys-1, demonstrated that met- 1 was linked to pyt -1 , and that arg-2 readily recombined with all the markers (Table 5). Cross 5, leu-1 ino-1 mor-1 ben-1 1 pyt-1 red-1 arg-2, pursued the relationship of arg- 2 to other markers; again it was not possible to demonstrate linkage, but the other markers behaved in a usual manner and four, red- 1 , ino- 1 , pyt- 1 and ben- 1 , all appeared tightly linked (Table 6). The most likely explanation is that the strains carried chromosome aberrations which involved three linkage groups; however, there is very little evidence from other crosses to support this hypothesis and the problem is still under investigation. Free recombination between $l e u-1$ and mor- 1 was demonstrated in cross 6 , leu-1 ino-1 mor-1 ben-1 $\times$ pyt-1 red-1 lys-1, completing the picture (Table 7).

In these and other three-factor crosses the leu-l allele continued to show a reduced fitness. All other crosses supported the linkage relationships reported (Hamlyn, 1982).

The final investigation, cross 7 , red-1 ino- 1 azu-1 ben-1 $\times$ lys-1 his-1, was done to determine the relationship between red-1, ino-1 and his-1 (Table 8). The behaviour of the red-1 allele on the medium which forced recombination between ino-l and his- 1 was particularly instructive. red-l appeared unlinked to ino- 1 (56\% recombination) and his-l $(43.5 \%$ recombination), a result which could only be explained if the gene order was ino-l red-1 his-1 and the relative recombination rates on either side of red-l were similar. This was supported by the results from the other medium with recombination rates of: his-1 to red $-1,3.9 \%$, red-1 to ino- $1,11.7 \%$; his-1 to ino- $1,15 \cdot 6 \%$.

Taking the results from all of the crosses it is possible to construct a tentative genetic map with the markers distributed between eight linkage groups: I, leu-1; II, met-1, pyt-1; III, his-1, red-1, ino-1; IV, arg-2; V, arg-1; VI, azu-1, lys-1; VII, mor-1; VIII, ane-5, ben-1.

\section{Heterozygotes}

During the analysis of the crosses it was found that a minority component of the selectants consisted of small abnormal unstable colonies which proved to be heterozygous for one or more markers. These segregants were certainly aneuploid for one or more chromosomes and some may have approached a diploid constitution. They were analysed using the classical techniques of parasexual genetics developed for diploid and aneuploid strains of Aspergillus nidulans (Roper, 1952; Upshall et al., 1979). One of the most interesting heterozygotes was isolated from cross 1 because it proved to be heterozygous for all of the marked linkage groups (Table 9). The results from the analysis of this heterozygote were very similar to those reported above. This was a very useful comparison since there was no deliberate selection for any particular genotypes. 
Table 4. Results from the analysis of selectants from cross 2 : azu-1 arg-1 mor-1 leu-1 red-1 pyt-1 ben-1 $\times$ ane-5 ino- 1

mor- 1 was not scored amongst these selectants. The pyt-l allele could not be scored in the presence of aneurin and was ignored amongst the selectants recovered from aneurin-containing media.

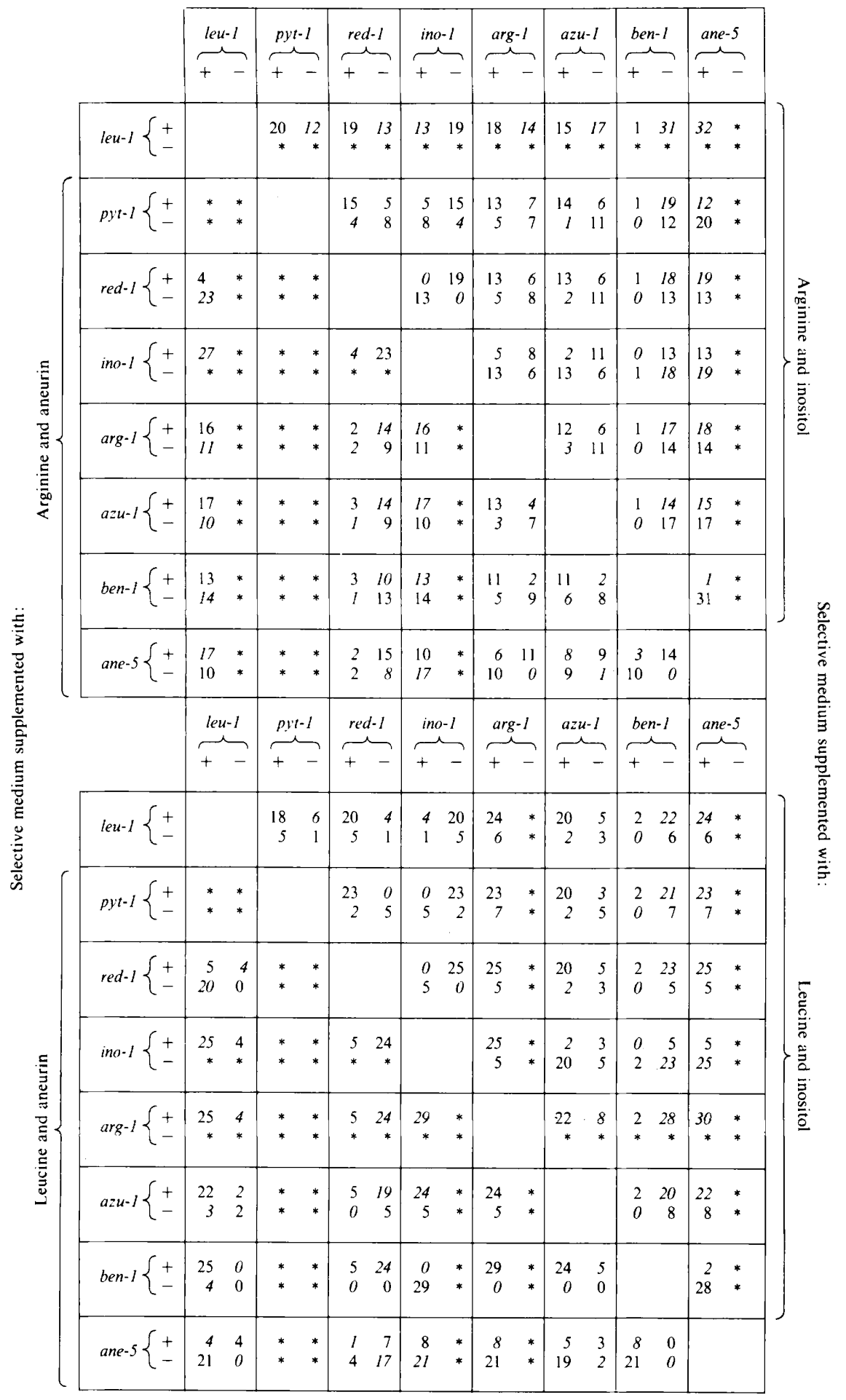


Table 5. Results from the analy sis of selectants from cross 4 : azu-I arg- 2 met-I $\times$ pyt-I red-1 lys- 1

$a z u-I$ was not scored amongst these segregants.

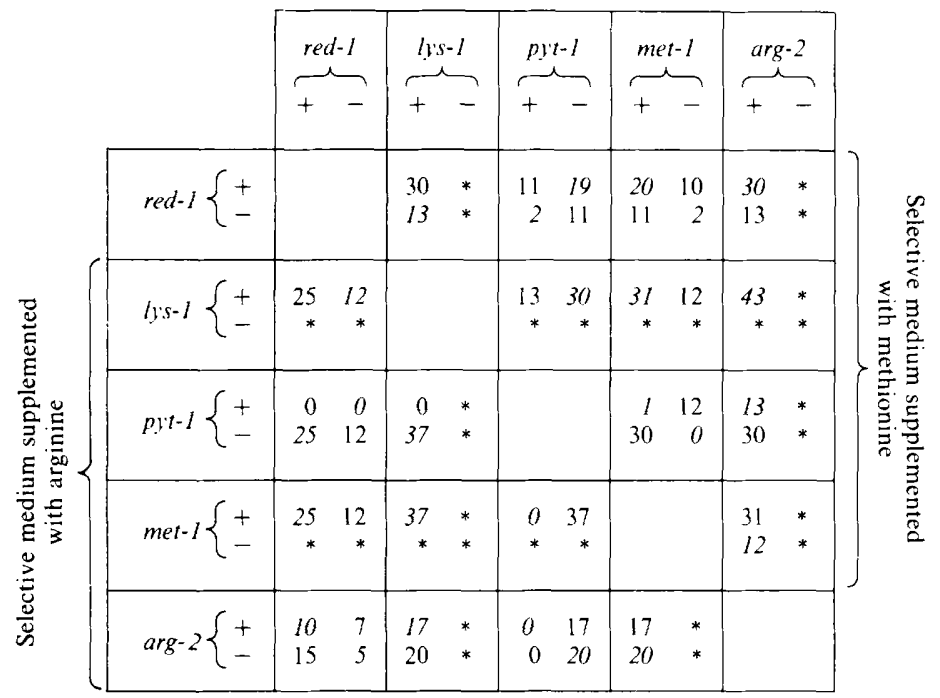

Table 6. Results from the analysis of selectants from cross 5 : leu-1 ino-1 mor-1 ben-1 $\times$ pyt-1 red-1 arg- 2

mor- $I$ was not scored amongst these segregants.

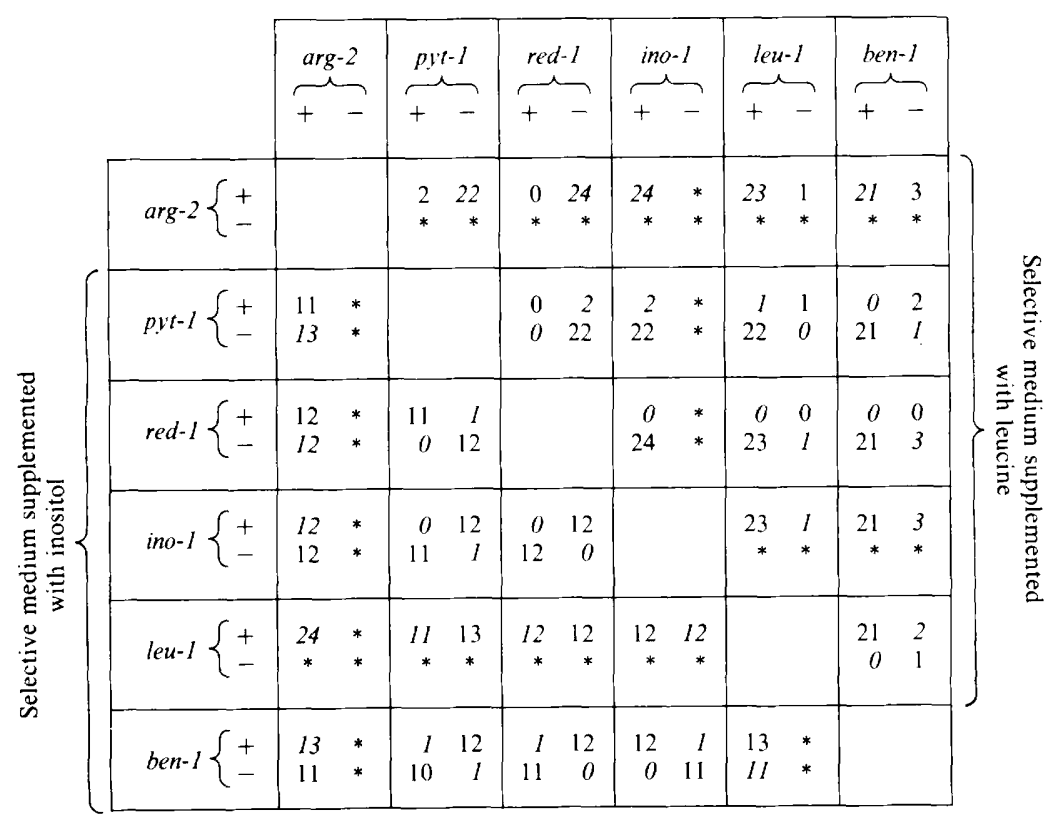


Table 7. Results from the analysis of selectants from cross 6 : leu-1 ino-1 mor-1 ben-1 $\times$ pyt-1 red-1 lys-1

pyt-l was not scored amongst these segregants.

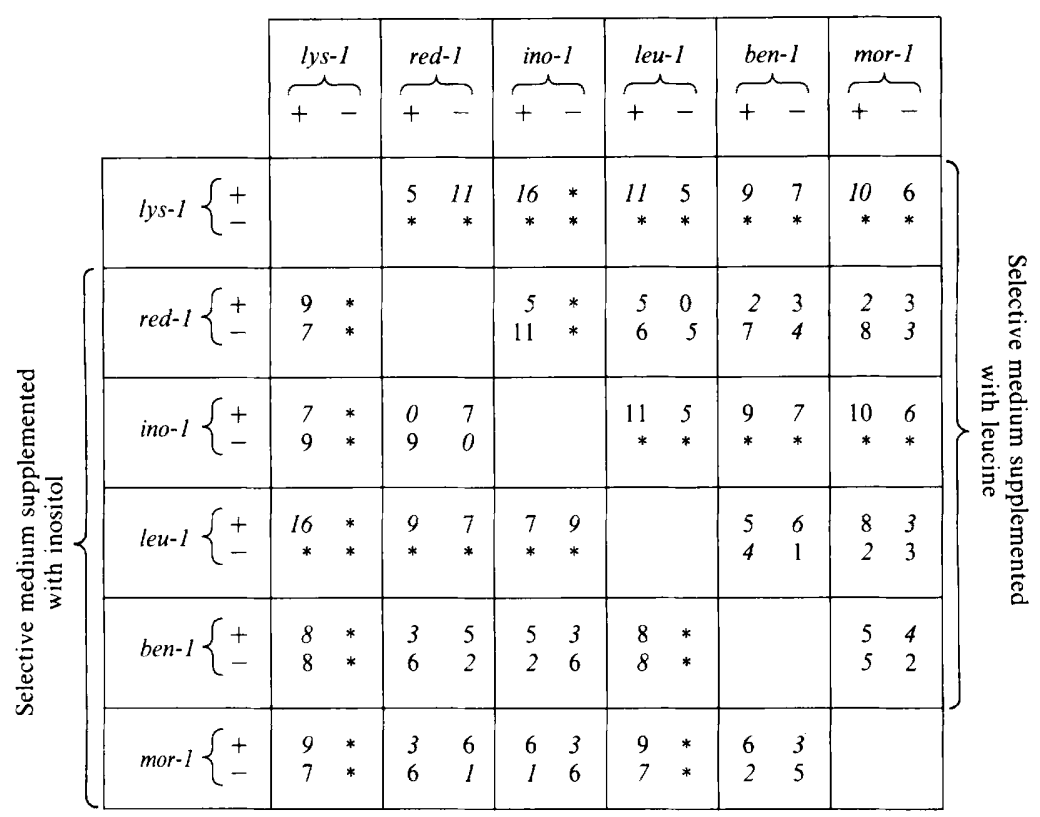

Table 8. Results from the analysis of selectants from cross 7 : red-1 ino-1 azu-1 ben-1 $\times$ lys-1 his-1

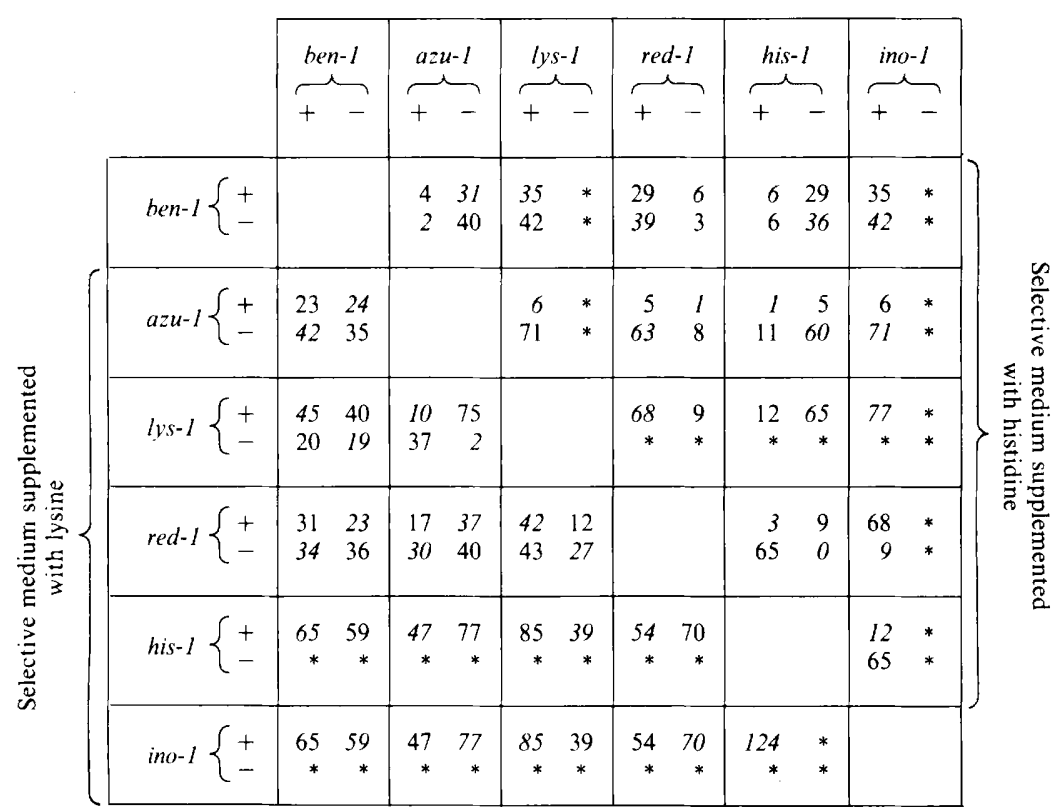


Table 9. Results from the analysis of the heterozygote isolated from cross 1: azu-1 arg-1 mor-1 leu-1 red-1 pyt-1 $\times$ lys-1 his-1

Segregants were recovered on CM.

\begin{tabular}{|c|c|c|c|c|c|c|c|}
\hline \multirow[b]{2}{*}{$\operatorname{leu-1}\left\{\begin{array}{l}+ \\
-\end{array}\right.$} & $\overbrace{+-}^{p y t-1}$ & $\overbrace{+\quad-}^{r e d-1}$ & $\overbrace{+\quad-}^{h i s-1}$ & $\overbrace{+\quad-}^{\text {arg-1 }}$ & $\overbrace{+-}^{\text {mor-1 }}$ & $\overbrace{+-}^{a z u-1}$ & $\overbrace{+\quad-}^{l y s-1}$ \\
\hline & $\begin{array}{rr}27 & 24 \\
4 & 1\end{array}$ & $\begin{array}{rr}17 & 34 \\
2 & 3\end{array}$ & $\begin{array}{rr}37 & 14 \\
1 & 4\end{array}$ & $\begin{array}{rr}38 & 13 \\
5 & 0\end{array}$ & $\begin{array}{rr}41 & 10 \\
4 & 1\end{array}$ & $\begin{array}{rr}16 & 35 \\
4 & 1\end{array}$ & $\begin{array}{rr}34 & 17 \\
1 & 4\end{array}$ \\
\hline & $p l y-1\left\{\begin{array}{l}+ \\
-\end{array}\right.$ & $\begin{array}{rr}10 & 21 \\
9 & 16\end{array}$ & $\begin{array}{rr}19 & 12 \\
19 & 6\end{array}$ & $\begin{array}{ll}24 & 7 \\
19 & 6\end{array}$ & $\begin{array}{ll}26 & 5 \\
19 & 6\end{array}$ & $\begin{array}{rl}15 & 16 \\
5 & 20\end{array}$ & $\begin{array}{rr}15 & 16 \\
20 & 5\end{array}$ \\
\hline & & $d-1\{+$ & $\begin{array}{rr}4 & 15 \\
34 & 3\end{array}$ & $\begin{array}{rr}16 & 3 \\
27 & 10\end{array}$ & $\begin{array}{ll}16 & 3 \\
29 & 8\end{array}$ & $\begin{array}{rr}2 & 17 \\
18 & 19\end{array}$ & $\begin{array}{rr}16 & 3 \\
19 & 18\end{array}$ \\
\hline & & & his- $\left\{\begin{array}{l}+ \\
-\end{array}\right.$ & $\begin{array}{rr}28 & 10 \\
15 & 3\end{array}$ & $\begin{array}{ll}31 & 7 \\
14 & 4\end{array}$ & $\begin{array}{rr}16 & 22 \\
4 & 14\end{array}$ & $\begin{array}{rr}21 & 17 \\
14 & 4\end{array}$ \\
\hline & & & & $\operatorname{rg}-1\left\{\begin{array}{l}+ \\
-\end{array}\right.$ & $\begin{array}{ll}34 & 9 \\
11 & 2\end{array}$ & $\begin{array}{rr}15 & 28 \\
5 & 8\end{array}$ & $\begin{array}{rr}27 & 16 \\
8 & 5\end{array}$ \\
\hline & & & & & $r-1\left\{\begin{array}{l}+ \\
-\end{array}\right.$ & $\begin{array}{rr}18 & 27 \\
2 & 9\end{array}$ & $\begin{array}{rr}25 & 20 \\
10 & 1\end{array}$ \\
\hline & & & & & & $c u-1\left\{\begin{array}{l}+ \\
-\end{array}\right.$ & $\begin{array}{rr}1 & 19 \\
34 & 2\end{array}$ \\
\hline
\end{tabular}

There was a general under-recovery of auxotrophic markers, with the leu-l allele being an extreme case. This indicated that the problems associated with the selective techniques were not solely caused by protoplast regeneration. Segregation of the heterozygote appeared to take place by the stepwise loss of chromosomes (Kafer, 1961). Several derivatives were isolated that had become homozygous or euploid for one or more markers whilst remaining heterozygous for the others.

One particular type of heterozygote was associated with the arg-2 allele; these carried the wild-type $A R G-2$ allele but only produced $\arg -2$ segregants, and their behaviour was probably associated with a chromosome aberration, as discussed by Hamlyn \& Ball (1979). Cross 7 yielded a segregant heterozygous for lys-1, red-1, ino-l and his-l. It was used to follow the segregation of the linkage group III markers. The results were similar to the previous data (Table 10) except that recombination frequencies appeared higher: his- 1 to red $-1,14 \cdot 1 \%$ red-l to ino- $1,23 \cdot 1 \%$; his- 1 to ino- $1,14 \cdot 1 \%$. This was undoubtedly due to clonal effects since one of the complementary genotypes appeared at a high frequency compared with its reciprocal type. Similar effects have been encountered during the analysis of other heterozygotes (Perez, 1984) and correcting for this distortion produced a set of recombination frequencies very similar to those from selective techniques: his-1 to red-1, 5.6\%; red-1 to ino- $1,15 \cdot 5 \%$; his-1 to ino- $1,15 \cdot 5 \%$. 
Table 10. Segregation of the linkage group III markers in two heterozygotes isolated from cross 7: red-1 ino-1 azu-1 ben-1 $\times$ lys-1 his-1

Segregants were recovered on CM.

\begin{tabular}{|c|c|c|c|c|c|}
\hline \multicolumn{3}{|c|}{ Genotype* } & \multicolumn{3}{|c|}{ No. of colonies } \\
\hline his-l & red-1 & ino- 1 & Isolate 1 & Isolate 2 & Total \\
\hline- & + & $t$ & 17 & 16 & 33 \\
\hline+ & - & - & 15 & 10 & 25 \\
\hline- & - & - & 0 & 0 & 0 \\
\hline+ & + & + & 1 & 1 & 2 \\
\hline- & + & - & 5 & 0 & 5 \\
\hline+ & - & + & 3 & 1 & 4 \\
\hline- & -- & + & 1 & 0 & 1 \\
\hline+ & + & - & 2 & 6 & 8 \\
\hline
\end{tabular}

\section{Post-fusion events}

Taking all the evidence into consideration it seems likely that the initial effect of protoplast fusion is to stimulate cytoplasmic mixing and then nuclear fusion at a frequency of 1 in $10^{4}$ to 1 in $10^{5}$ viable protoplasts. This diploid or polyploid condition results in unbalanced growth, the regenerating colonies being abnormal and slow growing. The growth advantage of any euploid non-disjunction products was so great that they rapidly overgrew the original colony. Because of this effect it was not possible to judge whether these nuclei underwent non-disjunction at a higher frequency than those of other fungi. The similarity in mitotic recombination frequencies found in the heterozygotes and direct selectants confirmed that the primary role of protoplast fusion was to initiate the parasexual process. There was no evidence of a stable diploid phase in C. acremonium, contrary to the claim of Nuesch et al. (1973). Heterozygous diploids from the related species Cephalosporium mycophilum and Emericellopsis salmosynnemata are also very unstable (Tuveson \& Coy, 1961 ; Fantini, 1962) supporting the idea that this is a general property of this group of fungi.

P.F.H and J.F.P. wish to thank Glaxochem Ltd for the financial support for this work. G.P. would like to thank the Spanish Ministry of Universities and Research for their support.

\section{REFERENCES}

AnNé, J. \& Peberdy, J. F. (1976). Induced fusion of fungal protoplasts following treatment with polyethylene glycol. Journal of General Microbiology 92 , 413-417.

Chang, L. T., Terasaka, D. T. \& Elander, R. P. (1982). Protoplast fusion in industrial fungi. Decelopments in Industrial Microbiology 23, 21-29.

Elander, R. P., Corum, C. J., De Valeria, H. \& WILGUS, R. M. (1976). Ultraviolet mutagenesis and cephalosporin synthesis in strains of Cephalosporium acremonium. In Second International Symposium on the Genetics of Industrial Microorganisms, pp. 253271. Edited by K. D. Macdonald. London: Academic Press.

FanTINI, A. A. (1962). Genetics and antibiotic production of Emericellopsis species. Genetics 47, 161-177.

FERENCZY, L. (1981). Microbial protoplast fusion. Symposia of the Society for General Microbiology 31, $1-34$.
Ferenczy, L., Kevel, F. \& Szegedi, M. (1975). High frequency fusion of fungal protoplasts. Experientia 31, 1028-1030.

Hamlyn, P. F. (1982). Protoplast fusion and genetic analysis in Cephalosporium acremonium. $\mathrm{PhD}$ thesis, University of Nottingham, UK.

HAMLYN, P. F. \& BALL, C. (1979). Recombination studies with Cephalosporium acremonium. In Genetics of Industrial Microorganisms, pp. 185-191. Edited by O. K. Sebek \& A. I. Laskin. Washington, DC: American Society for Microbiology.

Hamlyn, P. F., Bradshaw, R. E., Mellon, F. M., Santiago, C. M., Wilson, J. M. \& Peberdy, J. F. (1981). Efficient protoplast isolation from fungi using commercial enzymes. Enzyme and Microbial Technology 3, 321-325.

KAFER, E. (1961). The processes of spontaneous recombination in vegetative nuclei of Aspergillus nidulans. Genetics 46, 1581-1609. 
MinUth, W. \& ESSER, K. (1983). Intraspecific, interspecific and intergeneric recombination in $\beta$ lactam producing fungi via protoplast fusion. European Journal of Applied Microbiology and Biotechno$\log _{y} 18,38-46$.

Newton, G. G. F. \& Abraham, E. P. (1956). Isolation of cephalosporin $C$, a penicillin-like antibiotic containing $\mathrm{D}-\alpha$-aminoadipic acid. Biochemical Journal 62, 651-658.

Nuesch, J., Treichler, H. J. \& Liersch, M. (1973). The biosynthesis of cephalosporin C. In Genetics of Industrial Microorganisms, vol 2, pp. 309-334. Edited by Z. Vanek, Z. Hostalek \& J. Cudlin. Amsterdam: Elsevier Publishing Co.

Peberdy, J. F. (1979). Fungal protoplasts: isolation, reversion and fusion. Annual Review of Microbiology 33, 21-39.

Peberdy, J. F. (1980). Protoplast fusion - a tool for genetic manipulation and breeding in industrial microorganisms. Enzyme and Microbial Technology 2, 23-29.

Perez, G. (1984). Protoplast fusion and its consequences for cephalosporin $C$ production in Acremonium chrysogenum. PhD thesis, University of Nottingham. UK.

ROPER, J. A. (1952). Production of heterozygous diploids in filamentous fungi. Experientia 8, 14-15.

Tuveson, R. W. \& CoY, D. O. (1961). Heterokaryosis and somatic recombination in Cephalosporium mycophilum. Mycologia 53, 244-253.

Upshall, A., Giddings, B., TeOw, S. C. \& MortiMORE, I. D. (1979). Novel methods of genetic analysis in fungi. In Genetics of Industrial Microorganisms, pp. 197-204. Edited by O. K. Sebek \& A. I. Laskin. Washington, DC: American Society for Microbiology. 